\title{
Supersymmetric Proof of the Hirzebruch- Riemann-Roch Theorem for Non-Kähler Manifolds
}

Andrei V. SMILGA

SUBATECH, Université de Nantes, 4 rue Alfred Kastler, BP 20722, Nantes 44307, France

E-mail: smilga@subatech.in2p3.fr

Received November 10, 2011, in final form January 04, 2012; Published online January 08, 2012

http://dx.doi.org/10.3842/SIGMA.2012.003

Abstract. We present the proof of the HRR theorem for a generic complex compact manifold by evaluating the functional integral for the Witten index of the appropriate supersymmetric quantum mechanical system.

Key words: index; Dolbeault; supersymmetry

2010 Mathematics Subject Classification: 53C55; 53C80

\section{Introduction}

It is fairly clear now that the theoretical high-energy physics (at least, its part represented in the hep-th section of the Archive), being unfortunately deprived since a certain time of the experimental feedback, is gradually transforming into a branch of pure mathematics. The synthesis of these two sciences is fruitful for both of them: field theorists have absorbed and use a lot of mathematical techniques and methods, but also many physical concepts turned out to be very useful in treating pure mathematical problems. One can e.g. mention in this regard the paper [1] where the methods of quantum field theory were used to derive topological knot invariants.

Another distinguished example is the Atiyah-Singer theorem for the index of Dirac operator which was first proven by pure mathematical methods [2] and then a physical, in many respects more simple and clear proof of this theorem has been found [3]. This physical proof was based on the isomorphism of the classical complexes (de Rham complex, Dolbeault complex, Hirzebruch complex and Dirac complex) to certain supersymmetric quantum mechanical systems, with the indices of all these complexes being mapped to the Witten indices [4] of the proper SQM systems.

The Atiyah-Singer theorem was not, however, proven in these papers in its whole generality. Namely, the formula for the index of the Dolbeault operator was derived for Kähler manifolds but not for a generic complex manifold. In the present paper, we fill this gap.

Before going into details, let us briefly outline the structure of the proof.

- At the first step, we write down, following [5], the SQM system whose supercharges may be interpreted as exterior holomorphic derivative $\partial$ and its conjugate $\partial^{\dagger}$ (see equations (3.1) and (3.4) below).

- For Kähler manifolds, the functional integral for the index $I=\operatorname{Tr}\left\{(-1)^{F} e^{-\beta H}\right\}$ can be reduced in the semiclassical limit $\beta \rightarrow 0$ to the ordinary integral (4.3). In the Kähler case, this coincides with the integral representation (4.4) used usually by mathematicians.

- For the manifolds that are not Kähler (such that the Kähler form $\omega$ is not closed), but which satisfy the so called SKT condition, $\partial \bar{\partial} \omega=0$, the functional integral can as well be reduced to the ordinary integral (4.3). (The integral representation (4.3) for the Dolbeault

\footnotetext{
${ }^{1}$ On leave of absence from ITEP, Moscow, Russia.
} 
index of the SKT manifolds was derived earlier in [6] by purely mathematical methods.) In this case, the integrands in (4.3) and (4.4) do not coincide. One can show, however, that their difference is an exact form such that the integrals (4.3) and (4.4) are equal.

- For generic complex manifolds, a direct evaluation of the functional integral is difficult by the reasons explained below. One can notice, however, that the Dolbeault complex is equivalent to a Dirac complex involving extra torsions (in the Kähler case, the torsions are absent). We show then that one can actually unwind these torsions by deforming continuously the lagrangian in such a way that supersymmetry is kept at every value of the deformation parameter. The index is not changed under such a deformation and coincides thereby with the index of a certain torsionless Dirac operator. The functional integral for the latter can be reduced to an ordinary integral (4.10) by standard methods. One can proceed then as in the SKT case and observe that the integrands in equations (4.10) and (4.4) differ by an exact form.

\section{Dolbeault complex}

We remind here some basic facts and definition of the complex differential geometry.

We precede it with the following remark. As was mentioned above, theoretical high energy physics is merging now with mathematics, but at the moment this merge is far from being complete. As a result, there exist now two different communities with mathematical and physical backgrounds, studying in many cases very similar or just identical objects. Two communities use two different languages, such that a mathematical paper is more often than not incomprehensible to a physicist, and vice versa.

Speaking of this particular paper, it is written by a physicist and is addressed mainly to physicists even though its subject is in fact pure mathematical. Thus, I will use whenever possible the physical terminology even in the cases when a translation to mathematical language exists (and is known to the author). For example, we will not talk about line bundles and by no means about sheaves of germs, but only about Abelian gauge fields. In some cases, mathematical translations will be given in footnotes.

A complex manifold is a $2 n$-dimensional manifold covered by overlapping regions $U^{(a)}$, with every region being described by a set of complex coordinates $z^{j}, j=1, \ldots, n$ with the metric having Hermitian form

$$
g_{J K}=\left(\begin{array}{cc}
0 & h_{j \bar{k}} \\
h_{k \bar{j}} & 0
\end{array}\right) .
$$

The reality of the metric implies $\left(h_{j \bar{k}}\right)^{\star}=h_{k \bar{j}}$ such that $d s^{2}=2 h_{j \bar{k}} d z^{j} d \bar{z}^{\bar{k}}$. The coordinates $z^{j(a)}$ and $z^{j(b)}$ in the overlap $U^{(a)} \cap U^{(b)}$ are expressed into one another by holomorphic functions, $z^{j(a)}=f^{j(a b)}\left(z^{k(b)}\right)$. The Dolbeault complex is a set of all purely holomorphic forms,

$$
A^{(p)}=A_{j_{1} \ldots j_{p}} d z^{j_{1}} \wedge \cdots \wedge d z^{j_{p}}
$$

These forms should be regular on the manifold meaning that their norm is bounded

$$
\left\|A^{(p)}\right\|^{2}=A_{j_{1} \ldots j_{p}} A_{\bar{k}_{1} \ldots \bar{k}_{p}}^{\star} h^{\bar{k}_{1} j_{1}} \cdots h^{\bar{k}_{p} j_{p}}<\infty .
$$

Consider the operator of holomorphic exterior derivative, $\partial A^{(p)}=\partial_{k} A^{(p)} d z^{k} \wedge d z^{j_{1}} \wedge \cdots \wedge d z^{j_{p}}$ and its conjugate $\partial^{\dagger}$. The operators $\partial$ and $\partial^{\dagger}$ are nilpotent and can be interpreted as the supercharges $Q, \bar{Q}$. Their anticommutator $\left\{\partial, \partial^{\dagger}\right\}$ is called Dolbeault Laplacian and can be associated with the Hamiltonian. 
A Kähler manifold is the manifold where the metric $h_{j \bar{k}}$ satisfies the condition $\partial_{l} h_{j \bar{k}}=\partial_{j} h_{l \bar{k}}$ and can hence be derived from a Kähler potential, $h_{j \bar{k}}=\partial_{j} \partial_{\bar{k}} K$. Alternatively, one can say that the Kähler form $\omega=h_{j \bar{k}} d z^{j} \wedge d \bar{z}^{\bar{k}}$ is closed, $\partial \omega=\bar{\partial} \omega=0$. For Kähler manifolds, Dolbeault Laplacian coincides with the conventional Laplace-Beltrami operator. In a generic case, it does not.

Note also that, in the Kähler case, the "small" Dolbeault complex just described can be enlarged. The "large" Dolbeault complex involves all forms (not necessarily holomorphic) and, on top of $\partial$ and $\partial^{\dagger}$, also antiholomorphic exterior derivative $\bar{\partial}$ and its conjugate $\bar{\partial}^{\dagger}$. The SQM systems isomorphic to large Dolbeault complexes enjoy extended supersymmetry. They will not interest us in this paper.

Complex manifolds can be described using real notations if introducing the so called complex structure matrix $I_{M}^{N}, M \equiv\{m, \bar{m}\}$. It represents a tensor satisfying the conditions

$$
I_{M}^{N} I_{N}^{P}=-\delta_{M}^{P}, \quad I_{M}^{P} g_{P N}+I_{N}^{P} g_{P M} \equiv I_{M N}+I_{N M}=0,
$$

where $g_{M N}$ is the metric. Once complex coordinates $z^{j}, \bar{z}^{\bar{j}}$ and the Hermitian metric $h_{j \bar{k}}$ are defined, the complex structure matrix has only nonzero components with both holomorphic or both antiholomorphic indices, $I_{M}^{N}=\left\{i \delta_{m}^{n},-i \delta_{\bar{m}}^{\bar{n}}\right\} .^{2}$

Consider the standard Christoffel symbols

$$
\Gamma_{M N}^{P}=\frac{1}{2} g^{P Q}\left(\partial_{M} g_{P N}+\partial_{N} g_{P M}-\partial_{Q} g_{M N}\right)
$$

and the corresponding covariant derivative operator $\nabla_{M}$. Nonvanishing components of $\Gamma_{M N}^{P}$ for the Hermitian metric (2.1) are

$$
\begin{aligned}
& \Gamma_{m n}^{p}=\left(\Gamma_{\bar{m} \bar{n}}^{\bar{p}}\right)^{\star}=\frac{h^{\bar{q} p}}{2}\left(\partial_{m} h_{n \bar{q}}+\partial_{n} h_{m \bar{q}}\right), \\
& \Gamma_{n \bar{m}}^{\bar{p}}=\Gamma_{\bar{m} n}^{\bar{p}}=\left(\Gamma_{m \bar{n}}^{p}\right)^{\star}=\left(\Gamma_{\bar{n} m}^{p}\right)^{\star}=\frac{h^{\bar{p} q}}{2}\left(\partial_{n} h_{q \bar{m}}-\partial_{q} h_{n \bar{m}}\right) .
\end{aligned}
$$

If the metric is Kähler, the components of mixed holomorphicity vanish and only the components $\Gamma_{m n}^{p}$ and $\Gamma_{\bar{m} \bar{n}}^{\bar{p}}$ survive. This implies that the complex structure tensor is covariantly constant, $\nabla_{P} I_{M}^{N}=0$. (The condition $\nabla_{P} g_{M N}=0$ is also, of course, satisfied.)

Introduce the vielbeins $e_{M}^{A}, e_{A}^{M}$ (such that $e_{M}^{A} e_{B}^{M}=\delta_{B}^{A}$ and $e_{M}^{A} e_{N}^{A}=g_{M N}$ ) and consider the spin connection

$$
\Omega_{M, A}^{B}=e_{A N}\left(\partial_{M} e^{B N}+\Gamma_{M K}^{N} e^{B K}\right)
$$

and the corresponding 1-form $\Omega_{A}^{B}=\Omega_{M, A}{ }^{B} d x^{M}$. It satisfies the standard Maurer-Cartan structure equation

$$
d e_{A}+\Omega_{A}^{B} \wedge e_{B}=0
$$

For Kähler metrics, the only nonvanishing components of $\Omega_{A}^{B}$ are $\Omega_{a}^{b}$ and $\Omega_{\bar{a}}{ }^{\bar{b}}$. The same concerns the curvature matrix (whose elements represent 2-forms)

$$
R_{A}^{B}=d \Omega_{A}^{B}+\Omega_{A}^{C} \wedge \Omega_{C}^{B},
$$

\footnotetext{
${ }^{2}$ The inverse problem: to find complex coordinates once the complex structure matrix is given is nontrivial and does not always have a solution. The manifolds that are not complex but where the matrix $I_{M}^{N}$ with the properties (2.4) exists are called almost complex manifolds. For such manifolds, one cannot define a nilpotent holomorphic exterior derivative operator (there is no supersymmetry) and hence one cannot define Dolbeault complex and its index.
} 
where the mixed components $R_{a}{ }^{\bar{b}}$ and $R_{\bar{a}}{ }^{b}$ vanish. This means that the holomorphic tangent vectors stay holomorphic after a parallel transport along a closed contour: the holonomy group is $U(n)$.

If the manifold is not Kähler, $\nabla I \neq 0$, the mixed components in $\Omega_{A}^{B}$ and $R_{A}^{B}$ survive, and the holonomy group is $S O(2 n)$ as for a generic $2 n$-dimensional manifold. An important remark is, however, that one can make $I$ covariantly constant and reduce the holonomy group to $U(n)$ if allowing for nonzero torsions.

Consider an affine connection

$$
\gamma_{M N}^{P}=\Gamma_{M N}^{P}+K_{M N}^{P}
$$

where $\Gamma_{M N}^{P}$ is written in (2.5) and $K_{M N}^{P}$ is a tensor (its tensor nature will be important for us in the following) called contorsion tensor. For the metric to be still covariantly constant, $K_{Q, M N}=g_{P Q} K_{M N}^{P}$ should satisfy the condition $K_{Q, M N}=-K_{N, M Q}$. This implies a nonzero torsion

$$
T_{M N}^{P}=\gamma_{M N}^{P}-\gamma_{N M}^{P}=K_{M N}^{P}-K_{N M}^{P} \neq 0 .
$$

Let us impose an additional requirement for the tensor $C_{Q M N}=g_{P Q} T_{M N}^{P}$ to be totally antisymmetric. Then, for a complex manifold, there is a unique affine connection

$$
\hat{\Gamma}_{M N}^{P}=\Gamma_{M N}^{P}+\frac{1}{2} g^{P Q} C_{Q M N}
$$

satisfying $\hat{\nabla} g=\hat{\nabla} I=0 .{ }^{3}$ The components of $C_{Q M N}$ are expressed through the metric as

$$
\begin{aligned}
C_{j k \bar{l}} & =-C_{j \bar{l} k}=C_{\bar{l} j k}=\partial_{k} h_{j \bar{l}}-\partial_{j} h_{k \bar{l}}, \\
C_{\bar{j} \bar{k} l} & =-C_{\bar{j} l \bar{k}}=C_{l \bar{j} \bar{k}}=\left(C_{j k \bar{l}}\right)^{*}=\partial_{\bar{k}} h_{l \bar{j}}-\partial_{\bar{j}} h_{l \bar{k}} .
\end{aligned}
$$

The expressions (2.8) keep their form under holomorphic coordinate transformations. One can also represent the torsions in an arbitrary frame in real notations

$$
C_{Q M N}=I_{Q}^{P} I_{M}^{R} I_{N}^{T}\left(\nabla_{P} I_{R T}+\nabla_{R} I_{T P}+\nabla_{T} I_{P R}\right)
$$

such that the tensor nature of $C_{Q M N}$ is seen explicitly.

The connection (2.7), (2.9) arises naturally when a supersymmetric Lagrangian describing the Dolbeault complex on a generic complex manifold is built up [5]. Mathematicians know it as the Bismut connection. The corresponding Bismut spin connection (it is given by (2.6) with $\hat{\Gamma}$ substituted for $\Gamma$ ) and the Bismut curvature matrix do not have mixed components such that the holonomy group with this curvature is $U(n)$, as for the Kähler manifolds.

When the torsion is present, the Riemann tensor $R_{M N P Q}$ is not symmetric anymore with respect to interchange $\{M N\} \leftrightarrow\{P Q\}$. We have instead

$$
R_{M N P Q}(T)=R_{P Q M N}(-T) .
$$

\section{The SQM model}

Following [5], consider the chiral superfields

$$
Z^{j}=z^{j}+\sqrt{2} \theta \psi^{j}-i \theta \bar{\theta} \dot{z}^{j}, \quad \bar{Z}^{j}=\bar{z}^{\bar{j}}-\sqrt{2} \bar{\theta} \bar{\psi}^{j}+i \theta \bar{\theta} \dot{\bar{z}}^{\bar{j}}
$$

\footnotetext{
${ }^{3}$ There are many such connections if the condition $C_{Q M N}=C_{[Q M N]}$ is not imposed. One of them is the so called Chern or Hermitian connection with the only nonzero components $\Gamma_{\bar{p}, n m}^{C}=\left(\Gamma_{p, \bar{n} \bar{m}}^{C}\right)^{*}=\partial_{n} h_{m \bar{p}}$.
} 
$j, \bar{j}=1, \ldots, n, \bar{D} Z=D \bar{Z}=0$, and choose the supersymmetric action in the following form

$$
S=\int d t d^{2} \theta\left(-\frac{1}{4} h_{j \bar{k}}(Z, \bar{Z}) D Z^{j} \bar{D} \bar{Z}^{\bar{k}}+W(Z, \bar{Z})\right)
$$

with Hermitian $h_{j \bar{k}}$.

The component Lagrangian of this model can be cast in the following nice form

$$
\begin{aligned}
L= & \frac{1}{2}\left[g_{M N} \dot{x}^{M} \dot{x}^{N}+i g_{M N} \psi^{M} \hat{\nabla} \psi^{N}-\frac{1}{6} \partial_{P} C_{M N T} \psi^{P} \psi^{M} \psi^{N} \psi^{T}\right] \\
& +A_{M} \dot{x}^{M}-\frac{i}{2} F_{M N} \psi^{M} \psi^{N},
\end{aligned}
$$

where $x^{M} \equiv\left\{z^{j}, \bar{z}^{j}\right\}$, etc. and the torsion tensor $C_{M N T}$ is given in (2.8), (2.9). The second line in (3.2) involves the gauge potential $A_{M}=-I_{M}^{N} \partial_{N} W=\left(-i \partial_{m} W, i \partial_{\bar{m}} W\right)$ and its field strength $F_{M N}=\partial_{M} A_{N}-\partial_{N} A_{M}$.

The Lagrangian (3.2) is the Lagrangian of some particular supersymmetric sigma-model, its bosonic part describing free motion over the manifold. There are two complications here compared to the Kähler case: $(i)$ the covariant derivative $\hat{\nabla} \psi^{M}=\dot{\psi}^{M}+\hat{\Gamma}_{N K}^{M} \dot{x}^{N} \psi^{K}$ involves now the Bismut connection rather than usual torsion-free connection; (ii) a 4 -fermion term is present.

We will discuss the 4-fermion term a bit later, but let us first notice the appearance of the modified torsionfull connection. What is rather nontrivial and somewhat confusing is the fact that the supercharges also involve some modified connections, but this modification is not the same as in the Lagrangian!

The quantum covariant supercharges were presented in [5] in the form

$$
\begin{aligned}
& Q=\sqrt{2} \psi^{c} e_{c}^{k}\left[\Pi_{k}-\frac{i}{2} \partial_{k}(\ln \operatorname{det} \bar{e})-i \psi^{b} \bar{\psi}^{\bar{a}} \Omega_{k, b}{ }^{a}\right], \\
& \bar{Q}=\sqrt{2} \bar{\psi}^{\bar{c}} e_{\bar{c}}^{\bar{k}}\left[\bar{\Pi}_{\bar{k}}-\frac{i}{2} \partial_{\bar{k}}(\ln \operatorname{det} e)+i \bar{\psi}^{\bar{b}} \psi^{a} \bar{\Omega}_{\bar{k}, a}{ }^{b}\right],
\end{aligned}
$$

where $\operatorname{det} e$ and $\operatorname{det} \bar{e}$ are the determinants of the holomorphic and antiholomorphic vielbein matrices $e_{k}^{a}, e_{\bar{k}}^{\bar{a}}, \Pi_{k}=-i\left(\partial_{k}-\partial_{k} W\right)$, and $\bar{\Pi}_{k}=-i\left(\partial_{\bar{k}}+\partial_{\bar{k}} W\right)$. This expression involves the ordinary torsionless connections $\Omega$, but not all components of the latter, only the holomorphic ones $^{4}$.

When

$$
W=W_{0}=\frac{1}{2} \ln \operatorname{det} \bar{e}
$$

the operator $Q$ is isomorphic to the external holomorphic derivative $\partial$, and $\bar{Q}-$ to $\partial^{\dagger}$.

For other choices of $W$, we are dealing with the twisted Dolbeault complex involving an extra gauge field, $\partial X \rightarrow \partial X-i A^{\prime} \wedge X$ with $A^{\prime}=-i \partial_{j}\left(W-W_{0}\right) d z^{j}+i \partial_{\bar{j}}\left(W-W_{0}\right) d \bar{z}^{\bar{j}}$.

\footnotetext{
${ }^{4}$ The quantum supercharges and the quantum Hamiltonian (the expression for the latter was derived in [5], but we do not need it here) act on the holomorphic wave functions

$$
\Psi\left(z^{k}, \bar{z}^{k} ; \psi^{a}\right)=C^{(0)}\left(z^{k}, \bar{z}^{k}\right)+\psi^{j} C_{j}^{(1)}\left(z^{k}, \bar{z}^{k}\right)+\cdots+\psi^{j_{1}} \cdots \psi^{j_{n}} C_{j_{1} \ldots j_{n}}^{(n)}\left(z^{k}, \bar{z}^{k}\right)
$$

$\left(\psi^{j}=e_{a}^{j} \psi^{a}\right)$. The coefficients in this expansion are isomorphic to the forms (2.2).
} 
Remarkably, one can also derive $[7,8,9]$ that the sum $Q+\bar{Q}$ is isomorphic to the Dirac operator $i \not \nabla=i \tilde{\nabla}_{M} \gamma^{M}$ with $\tilde{\nabla}_{M}=\partial_{M}-i A_{M}+(1 / 4) \tilde{\Omega}_{M, A B} \gamma^{A} \gamma^{B}$ involving the gauge field $A=A^{\prime}+A^{(0)}$, where

$$
A_{M}^{(0)}=\frac{i}{2}\left\{-\partial_{m} \ln \operatorname{det} \bar{e}, \partial_{\bar{m}} \ln \operatorname{det} e\right\} .
$$

The potential (3.5) is gauge equivalent to $\frac{i}{4}\left\{-\partial_{m}, \partial_{\bar{m}}\right\} \ln \operatorname{det} h .^{5}$

Besides the gauge field, $\tilde{\nabla}_{M}$ involves also the spin connection

$$
\tilde{\Omega}_{M, A B}=\Omega_{M, A B}-\frac{1}{6} e_{A}^{L} e_{B}^{K} C_{M L K},
$$

where the torsions appear with the extra factor $1 / 3$ compared to the Bismut connection entering the Lagrangian. The difference $S=Q-\bar{Q}$ is isomorphic to the operator $\gamma^{M} I_{M}^{N} \tilde{\nabla}_{N}$.

The presence of the extra supercharge $S$ and hence the presence of the new supersymmetric structure $\{i \not \nabla, S\}$ in addition to the well-known chiral structure $\left\{i \not \nabla, \not \nabla \gamma^{D+1}\right\}\left(\gamma^{D+1}\right.$ being the multidimensional analog of $\gamma^{5}$ ) was noticed first in [10] for Kähler manifolds. But as we see now, this new structure is present for all complex manifolds with the only complication that the Dirac operator and the operator $S$ involve now the torsionfull connections (3.6).

\section{The index}

From now on, the manifold is assumed to be compact (for noncompact manifolds, the spectrum of the Hamiltonian is continuous and the notion of the index is ill-defined $)^{6}$. As we have just seen, the problem of calculating the index of the Dolbeault complex ${ }^{7}$ is reduced to the problem of calculating the index of the "nonstandard" Dirac operator involving extra torsions. The index of the ordinary Dirac operator was, of course, calculated by Atiyah and Singer and then in [3] using physical functional integral methods. In application to complex manifolds, this calculation was recently discussed in some details (the derivation in original papers was rather sketchy) in [5]. The Witten index of our SQM system is expressed via the path integral

$$
\begin{aligned}
I=\operatorname{Tr}\left\{(-1)^{F} e^{-\beta H}\right\}= & \lim _{N \rightarrow \infty} \int \prod_{\tau} \operatorname{det} h\left(\bar{z}^{\bar{j}}(\tau), z^{j}(\tau)\right) \prod_{j} \frac{d \bar{z}^{\bar{j}}(\tau) d z^{j}(\tau)}{2 \pi(\beta / N)} \\
& \times \prod_{a} d \psi^{a}(\tau) d \bar{\psi}^{\bar{a}}(\tau) \exp \left\{-\int_{0}^{\beta} L_{E}(\tau) d \tau\right\}
\end{aligned}
$$

where $L_{E}(\tau)$ is the Euclidean Lagrangian, $N$ is the number of points into which the Euclidean time interval $(0, \beta)$ is subdivided, and the periodic boundary conditions, $z(\beta)=z(0)$, $\psi(\beta)=\psi(0)$, are imposed onto all fields. The integral does not depend on $\beta$. To calculate it, we consider the semiclassical limit $\beta \ll 1$ when the integral is saturated by constant or nearly constant fields. For most systems, one can assume the fields to be constant, neglect higher Fourier harmonics and trade the functional integral for the ordinary one [12]. However, in this problem, such a simplified procedure does not work. One has to take into account higher harmonics which amounts to calculating loops.

\footnotetext{
${ }^{5}$ Mathematicians call the shift $A^{(0)}$ which appears when establishing this Dolbeault $\leftrightarrow$ Dirac correspondence the connection of the determinant bundle (to be quite precise, its square root).

${ }^{6}$ See, however, [11] where the Dolbeault complexes on $S^{4} \backslash\{\cdot\}$ and $S^{6} \backslash\{\cdot\}$ were studied. In spite of that $S^{4} \backslash\{\cdot\}$ is not compact, the spectrum of the Dolbeault Laplacian is still discrete, if including in the Hilbert space square integrable functions with the factor $\sqrt{g}=1 /\left(1+\bar{z}^{j} z^{j}\right)^{4}$ in the measure.

${ }^{7}$ Mathematicians sometimes call this index arithmetic genus.
} 
For Kähler metric, it is sufficient to perform a one-loop calculation. An accurate analysis (see [5] for details) shows that one loop contributions are of the same order as the tree level ones. Even though the former include a formally small factor $\beta$, but this factor is always multiplied by the structure $\bar{\psi}^{(0)} \psi^{(0)}\left(\psi^{(0)}\right.$ being the zero Fourier harmonic of the fermion field), which is of order of $1 / \beta$, as is seen from the tree level integral over zero harmonics. Now, for Kähler manifolds, one can show that the second and higher loop contributions are suppressed. The explicit one-loop calculation gives the result

$$
I=\int e^{\mathcal{F} / 2 \pi} \operatorname{det}^{-1 / 2}\left[\frac{\sin \frac{\mathcal{R}}{4 \pi}}{\frac{\mathcal{R}}{4 \pi}}\right],
$$

where $\mathcal{F}$ is the gauge field strength 2 -form and $\mathcal{R}$ is the matrix 2 -form associated with the Riemann tensor

$$
\mathcal{F}=\frac{1}{2} F_{M N} d x^{M} \wedge d x^{N}, \quad \mathcal{R}_{A}^{B}=\frac{1}{2} R_{A M N}^{B} d x^{M} \wedge d x^{N} .
$$

When expanding the integrand in (4.1) in Taylor series around unity, it represents a superposition of forms of different dimensions, but one has, of course, to pick up only the terms involving the top form $\propto d x^{1} \wedge \cdots \wedge d x^{2 n}$. Otherwise, the integral is zero. The determinant factor appearing in the integrand just indicates that we have performed a one-loop calculation with integrating over the higher Fourier modes.

In the generic complex case, the situation is substantially complicated by the presence of the 4 -fermion term in (3.2). As $\bar{\psi} \psi \sim 1 / \beta$, the integral of the 4 -fermion term is estimated to be $\int_{0}^{\beta}\left(\psi^{4}\right) d \tau \sim \beta / \beta^{2} \sim \beta^{-1}$. For small $\beta$, this contribution is large! Of course, being a total derivative, it does not contribute to the integral at the tree level but, after doing loop integrals, it could in principle be multiplied by some other structure and give a nonvanishing contribution. Actually, counting the powers of $\beta$ displays a worrisome fact that, to obtain a reliable result, we should perform in this case a honest two-loop calculation in 4 and 6 dimension, a honest three-loop calculation in 8 and 10 dimensions, etc.

Obviously, this calculation is not easy. A two-loop calculation for the heat kernel (the analog of the Kähler heat kernel $e^{\mathcal{F} / 2 \pi} \operatorname{det}^{-1 / 2}[\cdots]$ in equation (4.1)) of a 4-dimensional torsionfull Dirac operator was performed in [13] (and, indeed, this heat kernel involves rather intricate total derivative contributions). But nothing is known for higher dimensions.

Note, however, that there is a class of non-Kähler manifolds, namely, the manifolds where the form $\omega=h_{j \bar{k}} d z^{j} \wedge d \bar{z}^{\bar{k}}$ though not closed, satisfies the condition $\partial \bar{\partial} \omega=0$ such that the 4 -fermion term vanishes. These manifolds are called SKT manifolds ${ }^{8}$. It is known, for example, that all non-Kähler complex manifolds of complex dimension 2 (complex surfaces) belong to this class (more exactly, for any complex surface a SKT metric can always be chosen [14]). In this case, the functional integral for the Dolbeault index can be calculated by the same token as for the torsionless Dirac operator. We obtain

$$
I=\int e^{\left(\mathcal{F}^{\prime}+\mathcal{F}_{0}\right) / 2 \pi} \operatorname{det}^{-1 / 2}\left[\frac{\sin \frac{\hat{\mathcal{R}}}{4 \pi}}{\frac{\hat{\mathcal{R}}}{4 \pi}}\right],
$$

where $\mathcal{F}_{0}$ corresponds to the "geometrically induced" potential $(3.5), \mathcal{F}^{\prime}=d A^{\prime}$, with $A^{\prime}$ being the extra gauge field of the twisted Dolbeault complex, and $\hat{\mathcal{R}}$ is the curvature form of the Bismut connection that enters the Lagrangian (3.2). This formula was obtained in [6] by a rather refined mathematical reasoning. We see that the functional integral method allows one to derive it immediately for almost no price.

\footnotetext{
${ }^{8}$ SKT stands for strong Kähler with torsion. A little bit confusing because these manifolds are not Kähler, but, anyway, this is how they are called.
} 
However, the formula (4.3) for the Dolbeault index is not the way it was represented in $[15,2]$. They wrote instead

$$
I=\int e^{\mathcal{F}^{\prime} / 2 \pi} \operatorname{Td}(T M)
$$

where the symbol $\operatorname{Td}(T M)$ (Todd class of a complex tangent bundle associated with the manifold $M$ ) is spelled out as

$$
\operatorname{Td}(T M)=\prod_{\alpha=1}^{n} \frac{\lambda_{\alpha} / 2 \pi}{1-e^{-\lambda_{\alpha} / 2 \pi}},
$$

where $\lambda_{\alpha}$ are eigenvalues of the curvature matrix.

This is the Hirzebruch-Riemann-Roch theorem ${ }^{9}$.

One must say that there is a significant confusion associated with this formula in the physical literature. The curvature matrix is defined in (4.2). Were the elements of $\mathcal{R}_{A}{ }^{B}$ ordinary numbers, the eigenvalues can be found by diagonalizing

$$
\mathcal{R} \rightarrow \operatorname{diag}\left(i \lambda_{1} \sigma_{2}, \ldots, i \lambda_{n} \sigma_{2}\right)
$$

( $\sigma_{2}$ being a Pauli matrix). They are not numbers, however, but 2 -forms. Therefore, the eigenvalues $\lambda_{\alpha}$ do not have as such a lot of meaning and only their certain combinations (like $\left.\sum_{\alpha=1}^{n} \lambda_{\alpha}^{2}=\operatorname{Tr}\{\mathcal{R} \wedge \mathcal{R}\}\right)$ may have.

For Kähler manifolds, mixed holomorphic components $\mathcal{R}_{a}{ }^{\bar{b}}, \mathcal{R}_{\bar{a}}{ }^{b}$ vanish and one can consider instead of the antisymmetric $2 n \times 2 n$ matrix $\mathcal{R}_{A B}$, the $n \times n$ matrix $\mathcal{R}_{a}{ }^{b}$ and its eigenvalues ${ }^{10}$. The symmetric polynomials of the roots of the characteristic equation (having the order $n$ ), which enter equation (4.5), are expressed in a simple way into its coefficients and are reduced to combinations of products of the invariants $\operatorname{Tr}\{\mathcal{R}\}, \operatorname{Tr}\{\mathcal{R} \wedge R\}$, etc. But in a generic non-Kähler case, the characteristic equation has the order $2 n$. It involves only even powers of $\lambda$, such that its roots come in pairs $\left\{\lambda_{\alpha},-\lambda_{\alpha}\right\}$, and an expression like $\sum_{\alpha=1}^{n} \lambda_{\alpha}$ is simply meaningless!

This is true for the ordinary Riemann tensor that does not respect the holomorphic structure in the tangent space. We have learned, however, that even if the manifold is not Kähler, one can define the Bismut connection (2.7), (2.8) and the corresponding curvature tensor which $d o$ respect the holomorphic structure. The eigenvalues $\lambda_{\alpha}$ entering equation (4.5) refer to this Bismut torsionfull curvature tensor, not the usual one ${ }^{11}$ !

One can show that, in the Kähler case, the integrands in (4.3) and (4.4) coincide. Indeed, the factor $\operatorname{det}^{-1 / 2}[\cdots]$ in $(4.3)$ can be represented as $^{12}$

$$
\operatorname{det}^{-1 / 2}\left[\frac{\sin \frac{\mathcal{R}}{4 \pi}}{\frac{\mathcal{R}}{4 \pi}}\right]=\prod_{\alpha=1}^{n} \frac{\lambda_{\alpha} / 4 \pi}{\sinh \left(\lambda_{\alpha} / 4 \pi\right)},
$$

\footnotetext{
${ }^{9}$ It represents the multidimensional generalization of the Riemann-Roch theorem. The latter is a statement about the dimensions of the moduli spaces of meromorphic functions on Riemann surfaces, but this statement can be shown to be equivalent to the statement (4.4) about the Dolbeault index of Riemann surfaces equipped by Abelian gauge fields. (If trying to squeeze such a field on a single map, singularities (Dirac strings) appear. This singularities may be associated with the poles of meromorphic functions.) The HRR theorem was originally proven by Hirzebruch only for Kähler manifolds, but Atiyah and Singer proved it for all complex manifolds.

${ }^{10}$ Note that, with the convention (4.6) chosen, the eigenvalues of $\mathcal{R}_{a}{ }^{b}$ are not $\lambda_{\alpha}$, but $-i \lambda_{\alpha}$.

${ }^{11}$ To be more precise, one needs not to consider necessarily a Bismut connection, any connection satisfying $\nabla g=\nabla I=0$ is a complex tangent bundle connection and can be used to calculate the index. (To reiterate, the ordinary real tangent bundle connection (2.6) cannot be used for this purpose.) Even though the Todd polynomials (4.5) of different such complex connections are different, their integrals (4.4) are the same. We will see it soon.

${ }^{12}$ The r.h.s. of equation (4.7) is called A-roof genus.
} 
while

$$
e^{\mathcal{F}_{0} / 2 \pi} \stackrel{\text { Kähler }}{=} e^{i \mathcal{R}_{a}^{a} / 4 \pi}=\exp \left\{\frac{1}{4 \pi} \sum_{\alpha} \lambda_{\alpha}\right\} .
$$

The identity (4.7) is valid in a generic complex case (one has only to substitute $\hat{\mathcal{R}}$ for $\mathcal{R}$ ), but the relation (4.8) is not.

In the generic case

$$
i \hat{\mathcal{R}}_{a}^{a}=i d \hat{\Omega}_{a}^{a}=i d\left[\left(\partial_{\bar{j}} \ln \operatorname{det} e+\frac{1}{2} h^{\bar{k} t} C_{\bar{j} \bar{k} t}\right) d \bar{z}^{\bar{j}}-\left(\partial_{j} \ln \operatorname{det} \bar{e}+\frac{1}{2} h^{\bar{t} k} C_{j k \bar{t}}\right) d z^{j}\right],
$$

i.e. the connection (3.5) (that would lead alone to $\sum_{\alpha} \lambda_{\alpha}$ )is shifted by a certain vector.

Following the logics of [16], it is not difficult to see, however, that this shift does not affect the value of the integral. The variation of the integrand in (4.3) under an infinitesimal shift $A^{(0)} \rightarrow A^{(0)}+\epsilon B$ involves various terms. Consider one of them, say, the term

$$
X=\epsilon d B \wedge \mathcal{F}_{0} \wedge \hat{\mathcal{R}}_{a}^{b} \wedge \hat{\mathcal{R}}_{b}^{a} .
$$

Due to the facts $d \mathcal{F}_{0}=0$ and $d\left(\hat{\mathcal{R}}_{a}^{b} \wedge \hat{\mathcal{R}}_{b}{ }^{a}\right)=0$ (the latter identity is proven using the Bianchi identities

$$
d \hat{\mathcal{R}}_{a}^{b}-\hat{\mathcal{R}}_{a}^{C} \wedge \hat{\Omega}_{C}^{b}+\hat{\Omega}_{a}^{C} \wedge \hat{\mathcal{R}}_{C}^{b}=0
$$

and the fact that, for the Bismut connection, only the holomorphic dummy indices $C=c$ contribute), $X$ is an exact form and its integral is zero. The same reasoning can be applied to all other terms in the variation, like

$$
Y=\epsilon d B \wedge \mathcal{F}_{0} \wedge \mathcal{F}^{\prime} \wedge \mathcal{F}^{\prime} \wedge \operatorname{Tr} \hat{\mathcal{R}}^{4} \wedge \operatorname{Tr} \hat{\mathcal{R}}^{6}=\epsilon d\left(B \wedge \mathcal{F}_{0} \wedge \mathcal{F}^{\prime} \wedge \mathcal{F}^{\prime} \wedge \operatorname{Tr} \hat{\mathcal{R}}^{4} \wedge \operatorname{Tr} \hat{\mathcal{R}}^{6}\right)
$$

etc.

Consider now a finite shift $A(t)=A+t B$. We have just seen that the derivative of the integral over $t$ is zero. This means that $I(t)$ does not depend on $t$, i.e. it is the same for the shifted and unshifted gauge field.

This proof relies on the fact that $B$ is a regular 1-form well defined at every point of our manifold and satisfying the condition (2.3). Were it not so, for example, if $B \propto A$, the variation would still formally be an exact form, but it would involve singularities, and its integral might not vanish.

This proves the HRR theorem for the SKT manifolds. How to proceed in a general case when the 4-fermion term in (3.2) does not vanish and we cannot calculate the path integral?

We have seen that the problem of evaluating the Dolbeault index is reduced to the problem of evaluating the index of a nonstandard torsionfull Dirac operator. The point is that one can deform this nonstandard operator such that the torsions disappear. The index, however, is not changed under such smooth deformation. The invariance of a topological index under smooth deformation is a tool widely used in physics to calculate Witten indices of different supersymmetric theories [4]. It always works under the condition that supersymmetry survives under such deformation ${ }^{13}$.

\footnotetext{
${ }^{13}$ And if supersymmetry does not survive, it does not work. Torsions representing regular tensors on a manifold can be unwinded. But topologically nontrivial gauge fields cannot. Indeed, even if disregarding the fact that a correctly from mathematical viewpoint defined fiber bundle has always an integer topological charge and just trying to solve the Schrödinger equation for a system with a fractional topological charge (for example, a fractional magnetic flux on $S^{2}$ ), one would see that, for fractional charges, supersymmetry is lost [17]. And this is the reason why the deformation philosophy does not work in this case.
} 
Does the supersymmetry survive under an arbitrary torsion shift? After all, we have seen that the Dolbeault operator is isomorphic to the Dirac operator with some particular torsions given in (2.8). For other torsions, Dolbeault supersymmetry encoded in the superspace action (3.1) and associated with the existence of two supercharges $i \tilde{\nabla}_{M} \gamma^{M}$ and $S=\gamma^{M} I_{M}^{N} \tilde{\nabla}_{N}$ is lost. The point is, however, that an even-dimensional Dirac operator, irrespectively of whether it involves torsions or not, enjoys the supersymmetry associated with the chiral pair of supercharges $\left\{i \not \nabla\right.$ and $\left.\not \nabla \gamma^{D+1}\right\}$. The latter is true under two conditions: $i$ ) The manifold should admit spin structure ${ }^{14}$, which means that topological charge associated with the gauge field is quantized in a proper way (cf. the footnote remark above). ii) The torsions should represent a regular tensor. If either of these conditions is violated, the result of the action of $i \not \nabla$ on the regular spinors might be singular, and this breaks supersymmetry.

But in our case, the torsions (2.9) are regular tensors and the gauge field topological charge is quantized as it should. We then conclude that the Dolbeault index for an arbitrary compact complex manifold coincides with the index of the associated Dirac operator with unwinded torsions. This gives the following result

$$
I=\int e^{\mathcal{F}^{\prime} / 2 \pi} \exp \left\{\frac{1}{16 \pi} I_{M}^{P} \partial_{N} \partial_{P}(\ln \operatorname{det} g) d x^{M} \wedge d x^{N}\right\} \operatorname{det}^{-1 / 2}\left[\frac{\sin \frac{\mathcal{R}}{4 \pi}}{\frac{\mathcal{R}}{4 \pi}}\right],
$$

where we have explicitly written in real notations the contribution due to the metric-induced gauge field (3.5). The determinant factor involves the conventional torsionless Riemann tensor.

Let us show now that the integral (4.10) coincides with (4.4). Again, we can use the reasoning of $[16]$.

Consider the variation of the integrand in (4.10) under an infinitesimal deformation

$$
\omega_{A}^{B} \rightarrow \omega_{A}^{B}+\epsilon \lambda_{A}^{B}
$$

where $\lambda_{P M N}=e_{M}^{A} e_{B N} \lambda_{P, A}^{B}$ is a tensor. Then, say, the structure $\mathcal{R}_{A}^{B} \wedge \mathcal{R}_{B}^{A}$ is shifted as

$$
\delta\left(\mathcal{R}_{A}^{B} \wedge \mathcal{R}_{B}^{A}\right)=\epsilon\left(d \lambda_{A}^{B}+\lambda_{A}^{C} \wedge \Omega_{C}^{B}+\Omega_{A}^{C} \wedge \lambda_{C}^{B}\right) \wedge \mathcal{R}_{B}^{A}=\epsilon d\left(\lambda_{A}^{B} \wedge \mathcal{R}_{B}^{A}\right),
$$

where the Bianchi identity was used. The same reasoning applies to the terms like $\mathcal{R}_{A}^{B} \wedge \mathcal{R}_{B}^{C} \wedge$ $\mathcal{R}_{C}^{D} \wedge \mathcal{R}_{D}^{A}, \mathcal{F}^{\prime} \wedge \mathcal{F}^{\prime} \wedge \mathcal{R}_{A}^{B} \wedge \mathcal{R}_{B}^{A}$, etc. In other words, the variation is an exact form and its integral vanishes. This applies also to a finite deformation: we can deform (2.6) all the way to the Bismut connection and the integral for the index still does not change.

This brings the integral to the form (4.3). Then, following the procedure above, we deform further the gauge field (3.5) as in (4.9) and bring the integral to the canonical form (4.4). The theorem is proven.

\section{Example: Hopf manifolds}

The simplest example for non-Kähler complex manifolds are Hopf manifolds. Their metric has a simple form ${ }^{15}$

$$
d s^{2}=\frac{d \bar{z}_{j} d z_{j}}{\bar{z}_{k} z_{k}}
$$

and the complex coordinates $z_{j}$ lie in the region $1 \leq|z| \leq 2$, with the points $w_{j}$ and $2 w_{j}$ being identified $(|w|=1)$. The conformally invariant metric (5.1) is consistent with this identification.

\footnotetext{
${ }^{14}$ To be quite precise, spin $^{C}$ structure.

${ }^{15}$ In this section, we do not distinguish between covariant and contravariant, holomorphic and antiholomorphic indices.
} 
Topologically, the Hopf manifold $\mathcal{H}^{n}$ of complex dimension $n$ is a torus $S^{1} \times S^{2 n-1}$. The Kähler form $\omega_{n}$ associated with the metric (5.1) is not closed and $\mathcal{H}^{n}$ is not Kähler. Note, however, that $\partial \bar{\partial} \omega_{2}=0$ such that $\mathcal{H}^{2}$ represents (as all complex surfaces do [14]) an SKT manifold.

The (untwisted) Dolbeault Laplacian acting on the functions on $\mathcal{H}^{n}$ is

$$
-\frac{1}{2} \triangle^{\text {Dol }} \stackrel{\text { for } \stackrel{0-f o r m s}{=}}{=}-\left(\bar{z}_{k} z_{k}\right) \bar{\partial}_{j} \partial_{j}+z_{j} \partial_{j}
$$

to be compared with the usual Laplacian

$$
-\frac{1}{2} \triangle=-(\bar{z} z) \bar{\partial} \partial+\frac{1}{2}(z \partial+\bar{z} \bar{\partial}) .
$$

The operator (5.2) has one zero mode, $\Psi(\bar{z}, z)=1$. Actually, the operator $\triangle^{\text {Dol }}$ gives also zero, when acting on any antiholomorphic function $\chi(\bar{z})$. However, if $\chi(\bar{z})$ is not constant, it is not a function on $\mathcal{H}^{n}$ because it is not the same at $\bar{z}_{j}=\bar{w}_{j}$ and $\bar{z}_{j}=2 \bar{w}_{j}$

The full Dolbeault Laplacian has also a zero mode in the sector of $(1,0)$-forms. To see this, note that

$$
\triangle^{\mathrm{Dol}} \ln (\bar{z} z)=0 .
$$

$\ln (\bar{z} z)$ is not a function on $\mathcal{H}^{n}$, but the 1 -form

$$
P=\partial \ln (\bar{z} z)=\partial_{j}[\ln (\bar{z} z)] d z_{j}=\frac{\bar{z}_{j} d z_{j}}{\bar{z}_{k} z_{k}}
$$

is well defined on $\mathcal{H}^{n}$. It satisfies $\partial P=\partial^{\dagger} P=0$ and represents thus a zero mode of the full Dolbeault Laplacian ${ }^{16}$.

The index in this case is equal to $1-1=0$. It is instructive to reproduce this result by calculating the integrals (4.4) or, alternatively, (4.10) (the latter expression is somewhat simpler because it depends only on the ordinary curvature rather than the Bismut curvature). For the Hopf manifold, the symmetry dictates ${ }^{17}$

$$
\mathcal{R}_{M N}=A d x_{M} \wedge d x_{N}+B\left(x_{M} d x_{N}-x_{N} d x_{M}\right) \wedge\left(x_{Q} d x_{Q}\right) .
$$

In addition

$$
\mathcal{F}=\mathcal{F}_{0} \propto \partial_{j} \bar{\partial}_{k} \ln (\bar{z} z) d z_{j} \wedge d \bar{z}_{k}=\frac{d z_{j} \wedge d \bar{z}_{j}}{\bar{z} z}-\frac{\left(\bar{z}_{j} d z_{j}\right) \wedge\left(z_{k} d \bar{z}_{k}\right)}{(\bar{z} z)^{2}} .
$$

One can then be directly convinced that

$$
\mathcal{F} \wedge \mathcal{F}=0, \quad \mathcal{R}_{M N} \wedge \mathcal{R}_{N P}=0
$$

for any $A, B$.

The index represents a combination of the integrals of the terms like $\mathcal{F} \wedge \mathcal{F} \wedge \mathcal{R}_{M N} \wedge \mathcal{R}_{N P} \wedge$ $\mathcal{R}_{P Q} \wedge \mathcal{R}_{Q M}$ which all vanish due to (5.4).

\section{Acknowledgements}

I am indebted to G. Carron, E. Ivanov, L. Positselsky, S. Theisen, A. Wipf and, especially, M. Verbitsky for illuminating discussions and remarks. Special thanks are due to Alexei Rosly for carefully reading the manuscript and many valuable remarks.

\footnotetext{
${ }^{16}$ The property $\partial P=0$ is obvious. $\partial^{\dagger} P=0$ follows from (5.3). One can also check it directly using the map $d z_{j} \rightarrow \psi_{j}$ and the expressions (3.3) for the supercharges.

${ }^{17}$ The functions $A(x)$ and $B(x)$ can, of course, be calculated, but we do not need them.
} 


\section{References}

[1] Witten E., Quantum field theory and Jones polynomials, Comm. Math. Phys. 121 (1989), 351-399.

[2] Atiyah M.F., Singer I.M., The index of elliptic operators. I, Ann. of Math. (2) 87 (1968), 484-530. Atiyah M.F., Singer I.M., The index of elliptic operators. III, Ann. of Math. (2) 87 (1968), 546-604. Atiyah M.F., Singer I.M., The index of elliptic operators. IV, Ann. of Math. (2) 93 (1971), 119-138. Atiyah M.F., Singer I.M., The index of elliptic operators. V, Ann. of Math. (2) 93 (1971), 139-149.

[3] Alvarez-Gaumé L., Supersymmetry and the Atiyah-Singer index theorem, Comm. Math. Phys. 90 (1983), 161-173.

Friedan D., Windey P., Supersymmetric derivation of the Atiyah-Singer index and the chiral anomaly, Nuclear Phys. B 235 (1984), 395-416.

Windey P., Supersymmetric quantum mechanics and the Atiyah-Singer index theorem, Acta Phys. Polon. B 15 (1984), 435-452.

[4] Witten E., Dynamical breaking of supersymmetry, Nuclear Phys. B 188 (1981), 513-554.

Witten E., Constraints on supersymmetry breaking, Nuclear Phys. B 202 (1982), 253-316.

[5] Ivanov E.A., Smilga A.V., Dirac operator on complex manifolds and supersymmetric quantum mechanics, arXiv:1012.2069.

[6] Bismut J.-M., A local index theorem for non-Kähler manifolds, Math. Ann. 284 (1989), 681-699.

[7] Braden H.W., Sigma-models with torsion, Ann. Physics 171 (1986), 433-462.

[8] Mavromatos N.E., A note on the Atiyah-Singer index theorem for manifolds with totally antisymmetric $H$ torsion, J. Phys. A: Math. Gen. 21 (1988), 2279-2290.

[9] Fedoruk S.A., Ivanov E.A., Smilga A.V., Real and complex supersymmetric $d=1$ sigma models with torsion, in preparation.

[10] Kirchberg A., Länge J.D., Wipf A., Extended supersymmetries and the Dirac operator, Ann. Physics 315 (2005), 467-487, hep-th/0401134.

[11] Smilga A.V., Dolbeault complex on $S^{4} \backslash\{\cdot\}$ and $S^{6} \backslash\{\cdot\}$ through supersymmetric glasses, SIGMA 7 (2011), 105, 14 pages, arXiv:1105.3935.

[12] Cecotti S., Girardello L., Functional measure, topology and dynamical supersymmetry breaking, Phys. Lett. B 110 (1982), 39-43.

Girardello L., Imbimbo C., Mukhi S., On constant configurations and evaluation of the Witten index, Phys. Lett. $B 132$ (1983), 69-74.

[13] Obukhov Y.N., Spectral geometry of the Riemann-Cartan space-time, Nuclear Phys. B 212 (1983), $237-$ 254.

Peeters K., Waldron A., Spinors on manifolds with boundary: APS index theorem with torsion, J. High Energy Phys. 1999 (1999), no. 2, 024, 42 pages, hep-th/9901016.

[14] Gauduchon P., Le théorème de l'excentricité nulle, C. R. Acad. Sci. Paris Sér. A-B 285 (1977), A387-A390.

[15] Hirzebruch F., Arithmetic genera and the theorem of Riemann-Roch for algebraic varietes, Proc. Nat. Acad. Sci. USA 40 (1954), 110-114.

Hirzebruch F., Topological methods in algebraic geometry, Springer-Verlag, Berlin, 1978.

[16] Wu Y.S., Zee A., Massless fermions and Kaluza-Klein theory with torsion, J. Math. Phys. 25 (1984), 2696-2703.

[17] Smilga A.V., Non-integer flux: why it does not work, arXiv:1104.3986. 